\title{
ELEMENTARY INTRODUCTION TO REPRESENTABLE FUNCTORS AND HILBERT SCHEMES
}

\author{
STEIN ARILD STRØMME \\ Mathematical Institute, University of Bergen \\ Allégt. 55, N-5007 Bergen, Norway \\ E-mail: stromme@mi.uib.no
}

1. Introduction. The purpose of this paper is to define and prove the existence of the Hilbert scheme. This was originally done by Grothendieck in [4]. A simplified proof was given by Mumford [11], and we will basically follow that proof, with small modifications.

The present note started life as a handout supporting my lectures at a summer school on Hilbert schemes in Bayreuth 1991. I want to emphasize that there is nothing new in this paper, no new ideas, no new points of view.

To get an idea of a model for the Hilbert scheme, recall how the lines in the projective plane are in 1-1 correspondence with the points of another variety: the dual plane. Similarly, plane conic sections are parameterized by the 5 -dimensional projective space of ternary quadratic forms. More generally, hypersurfaces of degree $d$ in $\boldsymbol{P}^{n}$ are naturally parameterized by the projective space associated to $H^{0}\left(\boldsymbol{P}^{n}, \mathcal{O}_{\boldsymbol{P}^{n}}(d)\right)$. A generalization of the dual projective plane in another way: linear $r$-dimensional subspaces of $\boldsymbol{P}^{n}$ are parameterized by the Grassmann variety $G(r, n)$.

The Hilbert scheme provides a generalization of these examples to parameter spaces for arbitrary closed subschemes of a given variety. Consider the classification problem for algebraic space curves, for example. It consists of determining the set of all space curves, but also to deal with questions like which types of curves can specialize to which other types, or more generally, which algebraic families of space curves are there? (An algebraic family is a subscheme $Z \subseteq \boldsymbol{P}^{3} \times T$ where $T$ is a variety and each fiber $Z_{t}$ for $t \in T$ is a space curve. Thus $T$ serves as a parameter space for the family.)

A partial answer to the classification problem can be naively outlined as follows. Suppose we are working over the field $\boldsymbol{C}$ of complex numbers. Let $H$ be the set of all space curves, say of given degree $d$ and genus $g$. If $C \subseteq \boldsymbol{P}^{3}$ is such a curve, let $[C] \in H$ denote the corresponding point. For any variety $T$ and any subvariety $Z \subseteq \boldsymbol{P}^{3} \times T$ such that for each point $t \in T$, the fiber $Z_{t}$ is a curve in $\boldsymbol{P}^{3} \times\{t\} \simeq \boldsymbol{P}^{3}$ with the given degree and genus, we get a map of sets $\phi_{Z}: T \rightarrow H$ given by $\phi_{Z}(t)=\left[Z_{t}\right]$.

1991 Mathematics Subject Classification: 14-01, 14C05. 
Now suppose for a moment that we can give $H$ the structure of a complex algebraic variety, with this property that $\phi_{Z}$ is always a morphism of algebraic varieties. Assume furthermore that there exists a closed subscheme $W \subseteq \boldsymbol{P}^{3} \times H$ such that for each $h \in H$, the fiber $W_{h}$ is just the curve corresponding to $h$, in other words such that $\phi_{W}: H \rightarrow H$ is the identity morphism. If this is the case, then any morphism $T \rightarrow H$ would in turn give rise to a family $Z=W \times_{H} T \subseteq \boldsymbol{P}^{n} \times T$ such that $\phi_{Z}$ is exactly the given morphism $T \rightarrow H$.

Such a subscheme $W$ would be called a universal family of curves. The classification problem for curves of degree $d$ and genus $g$ would now be, at least in part, to describe in detail the parameter variety $H$ and the universal family $W$.

Actually, all this wishful thinking is almost true! But even if one is only interested in nonsingular space curves, one is forced to leave the category of varieties and work in the category of schemes instead. We also have to restrict our attention to flat families, a condition which is very natural if the parameter space of the family is a variety, slightly less trivial if it is a non-reduced scheme. Anyway, the resulting scheme $H$ is called the Hilbert scheme of space curves of degree $d$ and genus $g$, and if we allow all flat degenerations, it is a projective scheme, which is connected, but almost never smooth or irreducible.

The Hilbert scheme exists in much more general contexts than just space curves. The essential requirement seems to be that the ambient fixed space be projective, although there are other cases too. On the other hand, there exists an example, due to Nagata and Serre, of a complete nonsingular 3-dimensional variety $X$ such that even the Hilbert scheme parameterizing pairs of points in $X$ does not exist, see [4, p. 221-27].

From the preliminary definition of the Hilbert scheme above, it appears that it might be easier to understand the set of morphisms from other varieties into $H$ than to understand $H$ itself. These sets of morphisms form a functor in a natural way, and we therefore start with a section on functors and how to represent them by schemes.

The Hilbert functor, and hence the Hilbert scheme, is relatively easy to define. We shall construct the Hilbert scheme as a closed subscheme of a Grassmann variety, by essentially writing down its equations, at least in the case where the ambient space is $\boldsymbol{P}^{n}$. However, the Hilbert scheme is still somewhat indirectly given. For example, in most cases it is unpractical to compute explicitly how large the ambient Grassmannian needs to be, and then use the defining equations. To answer questions about geometrical properties of the Hilbert scheme, like dimension, singularities, irreducible components, cohomology etc. other methods are needed, and to some extent, available.

For example, Grothendieck proved that if $Z \subseteq X$ is a closed subscheme, and the Hilbert scheme of $X$ exists, then the Zariski tangent space of this Hilbert scheme at the point $[Z]$ is canonically isomorphic to $\operatorname{Hom}_{X}\left(\mathcal{I}_{Z}, \mathcal{O}_{Z}\right)$. He also gave a criterion for smoothness. There is also an estimate, coming from deformation theory, for the dimension of the Hilbert scheme at a given point. Covering this deformation theory will take us too far, so we are content with just stating some results here.

Notation. We follow the notation of [6]. In particular, we use Grothendieck's contravariant $\boldsymbol{P}$ : If $V$ is a vector space, the points of $\boldsymbol{P}(V)$ are the hyperplanes in $V$, or equivalently, the rank-1 quotients of $V$. There is an analogous notation for affine spaces: $\boldsymbol{V}(V)=\operatorname{Spec} \operatorname{Sym}(V)$ is the affine space whose underlying vector space is the dual of $V$. For Grassmannians, we use the convention that $\operatorname{Grass}^{r}(V)$ represents the rank- $r$ quotients of $V$, and that $\operatorname{Grass}_{r}(V)$ the set of $r$-dimensional subspaces of $V$. This is admittedly not 
in accordance with Grothendieck's own notation, but seems more natural in view of his notation for the Quot schemes and for the Hilbert schemes themselves.

If $\mathcal{F}$ is a coherent sheaf on a scheme $X$, we will sometimes abbreviate $H^{i}(X, \mathcal{F})$ to $H^{i}(\mathcal{F})$ and write $h^{i}(\mathcal{F})=\operatorname{dim} H^{i}(\mathcal{F})$.

All varieties and schemes are assumed to be noetherian, unless the contrary is explicitly stated. The important exception is the Hilbert scheme, which is a countable union of noetherian schemes, hence only locally noetherian.

\section{Functors.}

The functor of points of a scheme. We assume familiarity with the definition of categories and functors. If $\mathcal{C}$ is a category, we denote by $\mathcal{C}^{o}$ the opposite category, i.e. the objects are the same but all arrows are reversed. Thus a contravariant functor on $\mathcal{C}$ is the same as a covariant functor on $\mathcal{C}^{o}$. The category of sets and mappings is denoted by $\underline{\text { sets, }}$, and the category of schemes is schemes. If $S$ is a scheme, schemes $/ S$ is the category of $S$-schemes and $S$-morphisms. If $h$ is a contravariant functor on schemes $/ S$ with values in sets, we write

$$
h:(\underline{\text { schemes }} / S)^{o} \rightarrow \underline{\text { sets. }} .
$$

If $f: X \rightarrow Y$ and $y \in h(Y)$, we sometimes write $f^{*}(y) \in h(X)$ for the image of $y$ under the map $h(f): h(Y) \rightarrow h(X)$. Note that reference to the functor itself is suppressed under this convention.

An interesting contravariant functor on schemes is the following. Let $X$ be a scheme. For any scheme $T$, put

$$
h_{X}(T)=\{\text { morphisms } t: T \rightarrow X\} .
$$

Then $h_{X}$ is a contravariant functor on schemes with values in sets. It is called the functor of points of $X$. A morphism $t: T \rightarrow X$ is a point of $X$ with values in $T$, or just a $T$-valued point of $X$. If $T=\operatorname{Spec}(A)$, we also say an $A$-valued point. If $T=\operatorname{Spec}(K)$ where $K$ is an algebraically closed field, it is called a geometric point of $X$. In this case we write things like " $t \in X$ is a geometric point" and " $k(t)=K$ ".

Note that this usage of the word point differs from the usual notion of a point of a scheme (corresponding to prime ideals), but not too much in the case of geometric points on an algebraic variety.

There is a relative version as well: If $X$ is an $S$-scheme, we have its functor of points on the category of $S$-schemes:

$$
h_{X / S}(T)=\{S \text {-morphisms } t: T \rightarrow X\} .
$$

In this situation, there is induced a morphism $p: h_{X} \rightarrow h_{S}$. The datum of an $S$-scheme $T$ is an element $t \in h_{S}(T)$. Then the relative point functor is given on the $S$-scheme $t: T \rightarrow S$ by

$$
h_{X / S}(T)=p(T)^{-1}(t) \subseteq h_{X}(T)
$$

When it is clearly understood what the base $S$ is, we may sometimes suppress the $S$ from notation and just write $h_{X}$ instead of $h_{X / S}$. This abuse of notation will not do much harm, in particular if $S$ is the spectrum of a field, for example.

ExAmple 2.1. Let $S=\operatorname{Spec}(B)$ for a $\operatorname{ring} B$, and let $X=\operatorname{Spec}(A)$ where

$$
A=B\left[x_{1}, \ldots, x_{n}\right] /\left(f_{1}, \ldots, f_{r}\right) .
$$


Then if $R$ is a $B$-algebra, an $R$-valued point of $X / S$ is a $B$-algebra homomorphism $A \rightarrow R$, which corresponds exactly to a solution in $R^{n}$ of the simultaneous equations $f_{i}=0$.

EXAMPLE 2.2. The point functor of $S$ over itself: $h_{S / S}(T)$ is a one-point set for all $S$-schemes $T$.

ExAmple 2.3. The point functor of a subscheme. Assume the $X \subseteq S$ is a subscheme. Then for $t: T \rightarrow S$ an $S$-scheme, we have

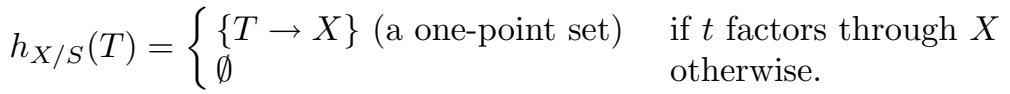

(Cf. Definition 2.11.)

EXAmple 2.4. The point functor of projective space. Let $X=\boldsymbol{P}_{k}^{n}$ and $S=\operatorname{Spec}(k)$, where $k$ is a field. Put $V=H^{0}\left(\boldsymbol{P}_{k}^{n}, \mathcal{O}_{\boldsymbol{P}^{n}}(1)\right)$. There is a canonical surjection $\phi: V_{\boldsymbol{P}^{n}} \rightarrow$ $\mathcal{O}_{\boldsymbol{P}^{n}}(1)$. Now for any $T$-point $t: T \rightarrow \boldsymbol{P}^{n}$ there is induced a surjection $t^{*} \phi: V_{T} \rightarrow$ $\phi^{*} \mathcal{O}_{\boldsymbol{P}^{n}}(1)$. Conversely, any linebundle quotient $V_{T} \rightarrow \mathcal{L}$ on $T$ gives rise to a unique morphism $T \rightarrow \boldsymbol{P}^{n}$. [6]. Hence

$$
h_{\boldsymbol{P}_{k}^{n} / k}(T)=\left\{\text { linebundle quotients of } V_{T}\right\},
$$

where two quotients are considered equal if they have the same kernel.

EXAmple 2.5. The Grassmann functor. Let $S=\operatorname{Spec}(k)$, and let $V$ be a vector space over $k$. Put $X=\operatorname{Grass}^{r}(V)$. Then

$$
h_{X}(T)=\left\{\text { rank- } r \text { locally free quotients of } V_{T}\right\} .
$$

EXAMPLE 2.6. Fiber products: $X^{\prime}=X \times_{Y} Y^{\prime}$ is actually defined by the property that $h_{X^{\prime}}=h_{X} \times_{h_{Y}} h_{Y^{\prime}}$. [6, 12]. In general, if $f_{1}, f_{2} \rightarrow g$ are morphisms of functors, we define the fiber product $f_{1} \times_{g} f_{2}$ to be the functor defined by the property that $\left(f_{1} \times_{g} f_{2}\right)(T)=f_{1}(T) \times_{g(T)} f_{2}(T)$ for all $T$.

Representable functors. Let $h:(\underline{\text { schemes }} / S)^{\circ} \rightarrow \underline{\text { sets }}$ be a functor. We want to know whether there exists an equivalence of functors $\psi: h_{X / S} \rightarrow h$ for some $S$-scheme $X$. For starters, any morphism of functors $\psi: h_{X / S} \rightarrow h$ gives rise to a canonical element $\xi=\psi\left(1_{X}\right) \in h(X)$. Conversely, let $X$ be an $S$-scheme and let $\xi \in h(X)$. Then there is induced a morphism $\psi_{\xi}: h_{X / S} \rightarrow h$ as follows: For any $t: T \rightarrow X$ in $h_{X / S}(T)$, put $\psi_{\xi}(t)=t^{*} \xi \in h(T)$. These constructions are inverses to each other, and gives a 1-1 correspondence between $h(X)$ and the set of morphisms $\psi: h_{X / S} \rightarrow h$.

Definition 2.7. The pair $(X, \xi)$ represents $h$ if the induced morphism $\psi_{\xi}: h_{X / S} \rightarrow h$ is an isomorphism. Equivalently, this can be formulated as follows: For any $T \rightarrow S$, there is a 1-1 correspondence

$$
\text { \{liftings } t: T \rightarrow X \text { of } T \rightarrow S\} \longleftrightarrow h(T)
$$

given by $t \leftrightarrow t^{*}(\xi)$. Note in particular that under the isomorphism $h \rightarrow h_{X / S}$, the identity $1_{X} \in h_{X / S}(X)$ corresponds to $\xi \in h(X)$.

A functor which can be represented like this is called representable, and the element $\xi$ is sometimes called the universal family. 
ExAmple 2.8. Coherent sheaves and vector bundles. Let $A$ be a $\operatorname{ring}$ and $M$ an $A$ module. Let $B=\operatorname{Sym}_{A}(M)$ be the symmetric algebra on $M$. There is a natural $A$-module homomorphism $i: M \rightarrow B$, identifying $M$ with the homogeneous part of degree 1 in the graded $A$-algebra $B$. By extension of scalars, we get a natural morphism $j: M \otimes_{A} B \rightarrow B$. This map is universal in the following sense: For any $A$-algebra $R$ and any $R$-module homomorphism $r: M \otimes_{A} R \rightarrow R$, there is a unique $A$-algebra morphism $f: B \rightarrow R$ such that $r$ is induced from $j$ via $f$. Put more briefly, there are natural bijections

$$
\operatorname{Hom}_{R}\left(M \otimes_{A} R, R\right)=\operatorname{Hom}_{A}(M, R)=\operatorname{Hom}_{A-\operatorname{algebras}}(B, R) .
$$

This is the affine version of the following:

Let $S$ be a scheme, and let $\mathcal{F}$ be a coherent sheaf on $S$. Consider the functor on schemes $/ S$ given by

$$
T \mapsto \operatorname{Hom}_{T}\left(\mathcal{F}_{T}, \mathcal{O}_{T}\right) .
$$

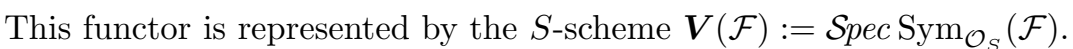

If $\mathcal{F}$ is locally free of rank $r$, then the symmetric algebra is locally isomorphic to a polynomial algebra, and $\boldsymbol{V}(\mathcal{F})$ is a vector bundle over $S$ in the geometric sense. Note the contravariant nature of this correspondence: If $\mathcal{F} \rightarrow \mathcal{G}$ is a morphism of sheaves, then there is an induced morphism $\boldsymbol{V}(\mathcal{G}) \rightarrow \boldsymbol{V}(\mathcal{F})$ of $S$-schemes.

EXAMPLE 2.9. Isomorphisms and principal bundles. Let $\mathcal{E}$ and $\mathcal{F}$ be vector bundles of the same rank $r$ on a scheme $S$, and consider the functor $\underline{\operatorname{Isom}}_{S}(\mathcal{E}, \mathcal{F})$ on $\underline{\text { schemes }} / S$ given by

$$
T \mapsto\left\{\text { set of } \mathcal{O}_{T} \text {-module isomorphisms } a: \mathcal{E}_{T} \rightarrow \mathcal{F}_{T} \cdot\right\}
$$

By the previous example, the functor of all homomorphisms $\mathcal{E}_{T} \rightarrow \mathcal{F}_{T}$ is represented by $V=\boldsymbol{V}\left(\mathcal{H}_{o} m_{\mathcal{O}_{S}}(\mathcal{F}, \mathcal{E})\right)$. There is a universal map $j: \mathcal{H o m}(\mathcal{F}, \mathcal{E})_{V} \rightarrow \mathcal{O}_{V}$, which corresponds to a universal homomorphism $b: \mathcal{E}_{V} \rightarrow \mathcal{F}_{V}$. Let $D \subseteq V$ be the hypersurface defined by the vanishing of the determinant of $b$. Then the $\operatorname{complement}_{\operatorname{Isom}}(\mathcal{E}, \mathcal{F}):=$ $V-D$ represents $\underline{\operatorname{Isom}}_{S}(\mathcal{E}, \mathcal{F})$.

In the special case where $\mathcal{F}=r \mathcal{O}_{S}$ is the trivial rank- $r$ vector bundle, $\operatorname{Isom}_{S}\left(\mathcal{E}, r \mathcal{O}_{S}\right)$ is called the principal homogeneous $G L(r)$-bundle associated to the vector bundle $\mathcal{E}$.

Digression on moduli spaces. Let $h$ be a given functor. Even if $h$ is not representable, it might be pretty close. The concept of a coarse moduli space rests on the idea of mapping $h$ to a representable functor $h_{M}$ rather than the other way around. More precisely, we have the following

Definition 2.10. A coarse moduli scheme for $h$ is a pair $(M, \lambda)$ where $M$ is a scheme and $\lambda: h \rightarrow h_{M}$ is a morphism of functors such that:

(1) For any algebraically closed field $k, \lambda(\operatorname{Spec}(k)): h(\operatorname{Spec}(k)) \rightarrow h_{M}(\operatorname{Spec}(k))$ is bijective, and

(2) If $\left(M^{\prime}, \lambda^{\prime}\right)$ is another pair satisfying (1), then there exists a unique morphism $f: M \rightarrow M^{\prime}$ such that $\lambda^{\prime}=h_{f} \circ \lambda$.

If a coarse moduli scheme $M$ for $h$ exists, then it is obviously unique. Furthermore, $h$ is representable $\Longleftrightarrow h$ is represented by $M \Longleftrightarrow$ there exists an element $\xi \in h(M)$ such that $\lambda(\xi)=1_{M}$. In this case, $M$ is called a fine moduli scheme for $h$. A coarse moduli functor is thus in a sense the best representable approximation to the given 
functor $h$. Putting it very roughly, in the "space" of all functors, $\lambda$ can be thought of as the "orthogonal projection" of $h$ onto the "subspace" of representable functors.

This should be contrasted with the concept of "formal moduli" or "pro-representable hull" of a functor which arises in infinitesimal deformation theory. In that case we look at functors on a slightly different (and smaller) category $\underline{\ell}$, and a pro-representable hull is then a morphism from a (pro-)representable functor to $h$, with certain properties $[13,8]$.

Subfunctors. If $Y \subseteq X$ is a subscheme, composition with the inclusion morphism gives rise to an inclusion $h_{Y}(T) \subseteq h_{X}(T)$ for all $T$. In fact, $h_{Y}$ is a subfunctor of $h_{X}$, in the following sense:

Definition 2.11. Let $g, h:(\underline{\text { schemes }} / S)^{\circ} \rightarrow$ sets. Then $g$ is a subfunctor of $f$ if $g(T) \subseteq$ $h(T)$ for all $T \rightarrow S$, and $g(t): g(T) \rightarrow g\left(T^{\prime}\right)$ is the restriction of $h(t)$ for all $S$-morphisms $t: T^{\prime} \rightarrow T$, i.e., the inclusion $g \rightarrow h$ is a natural transformation of functors. $g$ is said to be a closed (resp. open, resp. locally closed) subfunctor of $h$ if the following holds: For any $T \rightarrow S$ and $\xi \in h(T)$, there exists a closed (resp. open, resp. locally closed) subscheme $U_{\xi}^{g} \subseteq T$ such that for any $f: T^{\prime} \rightarrow T$, we have

$$
f^{*} \xi \in h\left(T^{\prime}\right) \text { belongs to the subset } g\left(T^{\prime}\right) \Longleftrightarrow f \text { factors through } U_{\xi}^{g} \subseteq T \text {. }
$$

An alternative way to formulate this is as follows: For any $S$-scheme $T$ and $\xi \in h(T)$, consider the cartesian square

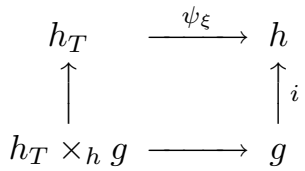

where $i$ is the inclusion. Then

Proposition 2.12. In this situation, $g$ is a closed (resp. open, resp. locally closed) subfunctor of $h$ if and only if for all $S$-schemes $T$ and elements $\xi \in h(T)$, the fiber product $h_{T / S} \times_{h} g$ induced by $\psi_{\xi}: h_{T / S} \rightarrow h$ is represented by a closed (resp. open, resp. locally closed) subscheme of $T$. In particular, if $h$ is representable and $g$ is a closed (resp. open, resp. locally closed) subfunctor of $h$, then $g$ is represented by a closed (resp. open, resp. locally closed) subscheme of the scheme representing $h$.

Pr o of. Put Definition 2.11 to work on the pair $(T, \xi)$ to produce a subscheme $U_{\xi}^{g} \subseteq T$. Then $h_{T / S} \times_{h} g$ is represented by $U_{\xi}^{g}$. For the second statement, let $T$ be the scheme representing $h$ and $\xi$ the universal family. Then $U_{\xi}^{g}$ represents $g$.

EXAMPLE 2.13. Let $\mathcal{F}$ be a coherent sheaf on $S$, let $r$ be an integer, and consider the subfunctor $h$ of $h_{S / S}$ (Example 2.2) defined by

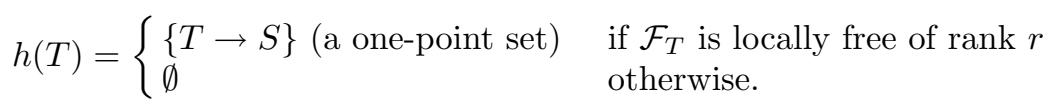

Then $h$ is a locally closed subfunctor of $h_{S / S}$, and it is represented by a locally closed subscheme $S_{r}(\mathcal{F}) \subseteq S$, see Corollary 6.3 below. 
Speculations. A scheme $X$ can be reconstructed from its functor of points $h_{X}$. Note that a morphism $X \rightarrow Y$ of schemes induces a morphism $h_{X} \rightarrow h_{Y}$ of contravariant functors. In fact, any morphism $h_{X} \rightarrow h_{Y}$ of functors is induced from a unique morphism of schemes $X \rightarrow Y$. Thus the association $X \mapsto h_{X}$ is an imbedding of the category of schemes as a full subcategory of the category sets ${ }^{(\text {schemes }}{ }^{\circ}$ of contravariant functors $\underline{\text { schemes }} \rightarrow \underline{\text { sets. }}$. All this is easy to prove, see [12].

It is also true that the restriction of $h_{X}$ to the subcategory of schemes consisting of affine schemes determines $X$ uniquely. One can even restrict $h_{X}$ to schemes of the form $\operatorname{Spec}(A)$ where $A$ is an Artin local ring, and still reconstruct much of the local structure of $X$. To give an example, suppose $S=\operatorname{Spec}(k), k$ an algebraically closed field, and let $t: \operatorname{Spec}(k) \rightarrow X$ be a $k$-point of $X$. Then the Zariski tangent space of $X$ at $t$ is nothing but the set of morphisms $\tilde{t}$ : $\operatorname{Spec}\left(k[\epsilon] / \epsilon^{2}\right) \rightarrow X$ "centered" in $t$, i.e., a certain subset of $h_{X / S}\left(\operatorname{Spec}\left(k[\epsilon] / \epsilon^{2}\right)\right)$

A related remark is this. If $f: X \rightarrow Y$ is a morphism of algebraic $k$-schemes, $k$ an algebraically closed field, then $f$ is scheme-theoretically injective (i.e. injective on points and tangentspaces) if and only if the induced morphism $h_{f}: h_{X} \rightarrow h_{Y}$ is injective in the sense that $h_{X}(T) \rightarrow h_{Y}(T)$ is injective for all $k$-schemes $T$. (It suffices to consider $T=\operatorname{Spec}(k)$ and $T=\operatorname{Spec}\left(k[\epsilon] / \epsilon^{2}\right)$.)

The moral here is first of all that the functor of points of a scheme contains a lot of information. In some sense, to understand a given scheme is to understand its point functor. (Not the only way to understand a scheme, but often the best!) On the other hand, when we consider the examples above, it is striking how easy it is to describe the functor of points of projective space, for example. It is much more complicated to give the data defining it as a scheme: affine open covers, sheaves of rings etc.

ExERCise 2.14. Let $X \rightarrow S$ be the blowing up of $S$ in some center $Y \subseteq S$. Try to understand the point functor $h_{X / S}$. Then explain why it is hard to understand blow-ups.

One is tempted to ask: If functors are so much better than schemes, then why not deal exclusively with functors and forget about schemes altogether? Well, one thing which is nice with schemes is that certain objects, like coherent sheaves, or morphisms, even the schemes themselves, may be constructed from compatible local data by gluing. For example, a representable functor $h$ is always a Zariski sheaf, meaning that for any scheme $T$ and any open cover $\left(T_{\alpha}\right)$ of $T$, the induced sequence

$$
h(T) \stackrel{f}{\longrightarrow} \prod_{\alpha} h\left(T_{\alpha}\right) \underset{g_{2}}{\stackrel{g_{1}}{\longrightarrow}} \prod_{\alpha, \beta} h\left(T_{\alpha} \cap T_{\beta}\right)
$$

is exact, meaning that $f$ is injective with image $\left\{x \mid g_{1}(x)=g_{2}(x)\right\}$.

Many properties of schemes and morphisms can be meaningfully defined also for functors.

Definition 2.15. Let $h:(\underline{\text { schemes }} / S)^{o} \rightarrow$ sets be a functor and $\left\{g_{i}\right\}_{i \in I}$ a collection of open subfunctors of $h$. We say that the $g_{i}$ form an open covering of $h$ if for all $S$-schemes $T$ and $\xi \in h(T)$, the sets $U_{\xi}^{g_{i}}$ of Definition 2.11 form an open cover of $T$.

Proposition 2.16. Let $h:(\underline{\text { schemes }} / S)^{\circ} \rightarrow \underline{\text { sets. }}$. If $h$ is a Zariski sheaf which admits an open covering by representable subfunctors, then $h$ is itself representable. 
Proof. Let $\left\{g_{i}\right\}_{i \in I}$ be a covering by representable, open subfunctors. Each $g_{i}$ is represented by a scheme $X_{i}$. Clearly the functor $g_{i} \times_{h} g_{j}$ is an open subfunctor of both $g_{i}$ and $g_{j}$, which allows us to glue the $X_{i}$ together to a scheme $X$. Both $h_{X}$ and $h$ are Zariski sheaves, and they coincide on an open covering. By the sheaf axiom, they must be isomorphic, hence $X$ represents $h$.

The Grassmannian. As an application, we prove that the Grassmann functor is representable:

Definition 2.17. Let $\mathcal{F}$ be a coherent sheaf over $S$, and let $r$ be an integer. The Grassmann functor $\underline{\operatorname{Grass}}^{r}(\mathcal{F})$ is the contravariant functor on schemes $/ S$ given by

$$
\underline{\operatorname{Grass}}^{r}(\mathcal{F})(T)=\left\{\text { rank- } r \text { locally free quotients of } \mathcal{F}_{T}\right\} \text {. }
$$

This functor is representable, but we prove it only in a special case here:

Proposition 2.18. Let $S=\operatorname{Spec}(A)$ and let $V$ be a free module of rank $n$ over $A$. Then $\operatorname{Grass}^{r}(V)$ is represented by a projective $S$-scheme $\operatorname{Grass}^{r}(V)$, which furthermore has an open cover of affine spaces over $S$ of dimension $r(n-r)$.

Proof. For each rank- $r$ free direct summand $W \subseteq V$, let $\underline{G}_{W}$ be the subfunctor of $\underline{\operatorname{Grass}}_{r}(V)$ corresponding to those quotients $V_{T} \rightarrow Q$ such that the induced map $W_{T} \rightarrow Q$ is an isomorphism. Choose a complement $K \subseteq V$ of $W$; then it is easy to see that $\underline{G}_{W}$ is represented by the affine space $G_{W}=\boldsymbol{V}\left(\operatorname{Hom}_{k}(K, W)^{*}\right)$ corresponding to the free module $\operatorname{Hom}_{A}(K, W)$ : an $A$-linear map $\phi: K \rightarrow W$ gives the quotient $\phi+1_{W}: K \oplus W \rightarrow W$.) It is clear that the Grassmann functor is a Zariski sheaf and that the $\underline{G}_{W}$ form an open covering as $W$ varies. Hence $\operatorname{Grass}^{r}(V)$ is represented by a scheme admitting a covering of affine spaces $G_{W}$ as claimed.

To show the projectivity assertion, let $V_{G} \rightarrow Q$ be the universal family. Define the Plücker morphism $\pi: G=\operatorname{Grass}_{r}(V) \rightarrow P=\boldsymbol{P}\left(\wedge^{r} V\right)$ as follows. The $r$-th exterior power yields a surjection $\wedge^{r} V_{G} \rightarrow \wedge^{r} Q$. This gives the required $G$-valued point of $P$. I claim that $\pi$ is a closed imbedding. It suffices to show that $P$ admits an affine open cover $\left\{P_{W}\right\}$ such that $\pi^{-1} P_{W} \rightarrow P_{W}$ is a closed imbedding for all $W$. To construct this, we again let $W$ run through the set of free rank- $r$ direct summands of $V$. For a fixed such $W \subseteq V$, we let $P_{W} \subseteq P$ be defined by the non-vanishing of the composed map $\wedge^{r} W_{P} \rightarrow \wedge^{r} V \rightarrow \mathcal{O}_{P}(1)$, i.e., $P_{W}$ is the complement of a hyperplane in $P$. For varying $W \subseteq V$, these $P_{W}$ form an open cover of $P$, and it is clear that $\pi^{-1} P_{W}=G_{W}$, the complement of the degeneration locus of the composed map $W_{G} \rightarrow V_{G} \rightarrow Q$.

To show that $\pi_{W}: G_{W} \rightarrow P_{W}$ is a closed imbedding, choose a complement $K$ of $W$ in $V$ as above. Then we have natural identifications

$$
\begin{aligned}
G_{W}(A) & =\operatorname{Hom}(K, W) \\
P_{W}(A) & =\operatorname{Hom}\left(\wedge^{r}(K \oplus W), \wedge^{r}(W)\right) / \wedge^{r}\left(0+1_{W}\right)=\bigoplus_{i=1}^{r} \operatorname{Hom}\left(\wedge^{i} K, \wedge^{i} W\right) \\
\pi_{W}(\phi) & =\left(\phi, \wedge^{2} \phi, \ldots, \wedge^{r} \phi\right)
\end{aligned}
$$

and hence the first component of the map is the identity. Therefore, $\pi_{W}$ is the graph morphism of the morphism $G_{W} \rightarrow \boldsymbol{V}\left(\bigoplus_{i=2}^{r} \operatorname{Hom}\left(\wedge^{i} K, \wedge^{i} W\right)^{\vee}\right)$ given by $\phi \mapsto\left(\wedge^{2} \phi, \ldots, \wedge^{r} \phi\right)$. The graph of a morphism into a separated scheme is closed, so $\pi_{W}$ is a closed imbedding.

See [7] for this and more general Grassmannians associated to vector bundles on a base scheme $S$. 
3. Definition of the Hilbert scheme. In this section we give the basic idea behind the construction of the Hilbert scheme. We make the definitions over an arbitrary (noetherian) base scheme $S$. Let $X$ be an $S$-scheme. If $T$ is another $S$-scheme, we make the following

Definition 3.1. An algebraic family of closed subschemes of $X / S$, parameterized by $T$, is a closed subscheme $Z \subseteq X_{T}=X \times_{S} T$. The family is flat if the induced morphism $Z \rightarrow T$ is flat. A geometric fiber of the family is the pullback $(1 \times t)^{*} Z$ of $Z$ to $X \times_{T}$ $\operatorname{Spec}(k(t))$, where $t: \operatorname{Spec}(k(t)) \rightarrow T$ is a geometric point of $Z$.

Definition 3.2. Let $\underline{\operatorname{Hilb}}_{X / S}(T)$ be the set of flat algebraic families of closed subschemes $Z$ of $X / S$ parameterized by $T$. If $T^{\prime} \rightarrow T$ is any morphism, $Z \mapsto Z \times_{T} T^{\prime}$ gives a map $\underline{\operatorname{Hilb}}_{X / S}(T) \rightarrow \underline{\operatorname{Hilb}}_{X / S}\left(T^{\prime}\right)$, which makes $\underline{\operatorname{Hilb}}_{X / S}$ a contravariant functor on the category of $S$-schemes.

If $\underline{H i l b}_{X / S}$ is representable, the $S$-scheme Hilb $_{X / S}$ representing it is called the Hilbert scheme of $X / S$.

Assume that $X \rightarrow S$ is projective, and choose a closed embedding $X \subseteq \boldsymbol{P}_{S}^{n}$. A numerical polynomial is a polynomial $P \in \boldsymbol{Q}[\mathrm{m}]$ such that $P(\ell) \in \boldsymbol{Z}$ for all $\ell \in \boldsymbol{Z}$. For any coherent sheaf $\mathcal{F}$ on $X$ and any geometric point $t$ of $T$, the induced sheaf $\mathcal{F}_{t}=(1 \times t)^{*} \mathcal{F}$ on $\boldsymbol{P}_{k(t)}^{n}$ has a Hilbert polynomial $P_{\mathcal{F}_{t}}$. If $Z$ is a flat familiy of closed subschemes of $X / S$ parameterized by a connected scheme $T$, then for all geometric points $t$ of $T$, the Hilbert polynomials $P_{\mathcal{F}_{t}}$ are the same [6]. Hence, if the Hilbert scheme exists, it necessarily decomposes into disjoint open and closed subschemes indexed by Hilbert polynomials. Therefore we make the following definition.

Definition 3.3. If $P$ is a numerical polynomial, let $\underline{\operatorname{Hilb}}_{X / S}^{P}$ be the open and closed subfunctor of $\underline{H i l b}_{X / S}$ given by flat families with Hilbert polynomial $P$ in all geometric fibers.

It is clear that if $\underline{\operatorname{Hilb}}_{X / S}^{P}$ is represented by some scheme $\operatorname{Hilb}_{X / S}^{P}$ for each $P$, then $\underline{\text { Hilb }}_{X / S}$ is represented by the disjoint union of all these.

The main body of the rest of the paper is devoted to proving that $\operatorname{Hilb}_{X / S}^{P}$ exists, provided that $X$ is projective over $S$. We start by giving the main idea. Consider the case where $S=\operatorname{Spec}(k)$. We want to study the set of all closed subschemes of $Z \subseteq X$ with Hilbert polynomial $P$. Let $Z$ be such a subscheme. Then there exists an $m_{0}$ such that $\mathcal{I}_{Z}(m)$ is generated by global sections and $H^{i}\left(X, \mathcal{I}_{Z}(m)\right)=0$ for all $i>0$ and $m \geq m_{0}$. In particular, $H^{0}\left(\mathcal{O}_{Z}(m)\right)$ is a quotient of $H^{0}\left(\mathcal{O}_{X}(m)\right)$ of rank $P(m)$ for all $m \geq m_{0}$, so $Z$ gives rise to a point in $\operatorname{Grass}^{P(m)}\left(H^{0}\left(\mathcal{O}_{X}(m)\right)\right)$, called the $m$-th Hilbert point of $Z$. Since $\mathcal{I}_{Z}(m)$ is generated by its global sections, the subscheme $Z$ is determined by its $m$-th Hilbert point, for any sufficiently large $m$.

This suggests that the Hilbert scheme might be taken to be a subscheme of $\operatorname{Grass}^{P(m)}\left(H^{0}\left(\mathcal{O}_{X}(m)\right)\right)$ for some sufficiently large $m$. Indeed, this is how it works, but there are essentially two difficulties to overcome. The first is to prove that one can find a value of $m_{0}$ which works for all subschemes at once (with the given Hilbert polynomial). This problem is treated in Section 4 , and uses the concept of $m$-regularity. Given its positive solution, we get an injective morphism from the Hilbert functor to the Grassmann functor. The second difficulty is to prove that this morphism is actually a locally 
closed imbedding. This requires some understanding of the flatness condition, and uses the concept of flattening stratification, covered in Section 7.

4. Boundedness. Throughout this section, we work over a field $k$. Write $\boldsymbol{P}^{n}=\boldsymbol{P}_{k}^{n}$. Following [11], we make the following definition:

Definition 4.1. A coherent sheaf $\mathcal{F}$ on $\boldsymbol{P}^{n}$ is $m$-regular if $H^{i}(\mathcal{F}(m-i))=0$ for all $i>0$.

Proposition 4.2 (Mumford-Castelnuovo). Let $\mathcal{F}$ be an $m$-regular sheaf on $\boldsymbol{P}^{n}$. Then

(1) $H^{0}(\mathcal{F}(k)) \otimes H^{0}\left(\mathcal{O}_{\boldsymbol{P}^{n}}(1)\right) \rightarrow H^{0}(\mathcal{F}(k+1))$ is surjective for $k \geq m$.

(2) $H^{i}(\mathcal{F}(k))=0$ whenever $k+i \geq m$ and $i>0$. In other words, $\mathcal{F}$ is $m^{\prime}$-regular for all $m^{\prime} \geq m$.

(3) $\mathcal{F}(k)$ is generated by global sections if $k \geq m$.

Proof. (Almost verbatim after [11].) We prove (1) and (2) together by induction on $n$, the case $n=0$ being trivial. If $n>0$, let $H \subseteq \boldsymbol{P}^{n}$ be a general hyperplane. Then there is an exact sequence

$$
0 \rightarrow \mathcal{F}(k-1) \rightarrow \mathcal{F}(k) \rightarrow \mathcal{F}_{H}(k) \rightarrow 0
$$

From the long cohomology sequence we get in particular:

$$
H^{i}(\mathcal{F}(m-i)) \rightarrow H^{i}\left(\mathcal{F}_{H}(m-i)\right) \rightarrow H^{i+1}(\mathcal{F}(m-i-1)),
$$

from which it follows immediately that $\mathcal{F}_{H}$ is $m$-regular. By induction, (1) and (2) hold for $\mathcal{F}_{H}$. Now consider the exact sequence

$$
H^{i}(\mathcal{F}(m-i)) \rightarrow H^{i}(\mathcal{F}(m-i+1)) \rightarrow H^{i}\left(\mathcal{F}_{H}(m-i+1)\right)
$$

If $i>0$, then by (2) for $\mathcal{F}_{H}$, the last group is zero, and the first group is also zero by assumption. It follows that $\mathcal{F}$ is $(m+1)$-regular. Continuing this way, $(2)$ is proved for $\mathcal{F}$. To get $(1)$, consider the diagram

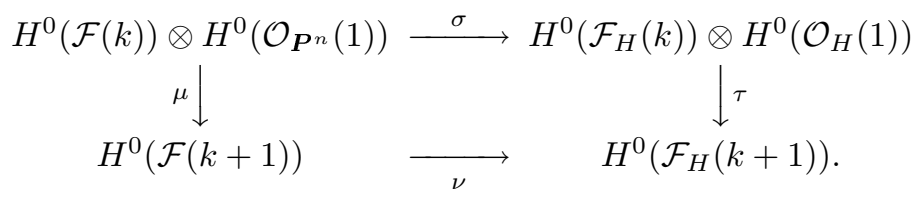

Note that $\sigma$ is surjective for $k \geq m$ because $H^{1}(\mathcal{F}(k-1))=0$ by (2). Moreover, $\tau$ is surjective if $k \geq m$ by (1) for $\mathcal{F}_{H}$. Therefore, $\nu \mu$ is surjective. Since clearly $\operatorname{Ker}(\nu) \subseteq$ $\operatorname{Im}(\mu)$, it follows that also $\mu$ is surjective, and (1) is proven for $\mathcal{F}$.

For $(3)$, we know by Serre's theorem $[6]$ that $\mathcal{F}(k)$ is generated by its global sections for $k \gg 0$. But by (1), these sections can all be expressed in terms of global sections of $\mathcal{F}(m)$. Hence these sections already generate $\mathcal{F}(m)$.

Proposition 4.3. Let $P$ be a numerical polynomial. Then there exists an integer $m_{0}=m_{0}(P)$ such that for any closed subscheme $Z \subseteq \boldsymbol{P}^{n}$ with Hilbert polynomial $P$, the ideal sheaf $\mathcal{I}_{Z}$ is $m_{0}$-regular.

Proof. Write $\mathcal{I}_{Z}=\mathcal{I}$. Again we use induction on $n$, the case $n=0$ being trivial. If $n>0$, let $H$ be a general hyperplane, giving rise to an exact sequence

$$
0 \rightarrow \mathcal{I}(-1) \rightarrow \mathcal{I} \rightarrow \mathcal{I}_{H} \rightarrow 0,
$$


where also $\mathcal{I}_{H} \subseteq \mathcal{O}_{H}$ is an ideal sheaf. By induction, there exists an integer $m_{1}$, depending only on $P$, such that $\mathcal{I}_{H}$ is $m_{1}$-regular. From the cohomology sequences we get immediately that $H^{i}(\mathcal{I}(k))=H^{i}(\mathcal{I}(k+1))$ as soon as $k \geq m_{1}-i$ and $i \geq 2$. By Serre's theorem, these groups must vanish. Hence $\mathcal{I}$ is also $m_{1}$-regular except possibly for the vanishing of $H^{1}\left(\mathcal{I}\left(m_{1}-1\right)\right)$. But we can control this using the following

Lemma 4.4. The sequence $\left\{h^{1}(\mathcal{I}(m))\right\}_{m \geq m_{1}-1}$ decreases strictly to zero.

Proof. The following sequence, exact for $m \geq m_{1}-1$ :

$$
H^{0}(\mathcal{I}(m+1)) \stackrel{\rho_{m}}{\longrightarrow} H^{0}\left(\mathcal{I}_{H}(m+1)\right) \rightarrow H^{1}(\mathcal{I}(m)) \rightarrow H^{1}(\mathcal{I}(m+1)) \rightarrow 0,
$$

shows that the sequence of the lemma is at least weakly decreasing. If for some $m$ we have $h^{1}(\mathcal{I}(m))=h^{1}(\mathcal{I}(m+1))$, then $\rho_{m}$ is surjective. But it is straightforward to show that $\rho_{m}$ surjective implies that $\rho_{m+1}$ is surjective as well. It follows that $h^{1}(\mathcal{I}(m))=$ $h^{1}(\mathcal{I}(m+1))=h^{1}(\mathcal{I}(m+2))$ etc. By Serre's theorem, these are all zero, and the lemma is proved.

It follows from this lemma that $H^{1}(\mathcal{I}(k))=0$ for $k \geq m_{1}-1+h^{1}\left(\mathcal{I}\left(m_{1}-1\right)\right)$. Hence if we let $m_{0} \geq m_{1}+h^{1}\left(\mathcal{I}\left(m_{1}-1\right)\right)$, then $\mathcal{I}$ is $m_{0}$-regular. It remains only to check that an $m_{0}$ can be found which depends only on $P$. But this is clear: since $H^{0}\left(\mathcal{O}_{Z}\left(m_{1}-1\right)\right)$ surjects onto $H^{1}\left(\mathcal{I}\left(m_{1}-1\right)\right)$, and the higher cohomology groups of $\mathcal{O}_{Z}\left(m_{1}-1\right)$ vanish by what we have already, it suffices to take $m_{0}=m_{1}+P\left(m_{1}-1\right)$. This completes the proof of Proposition 4.3.

Rem ark 4.5. If one analyzes this proof a little, one can show that $m_{0}$ can be taken to depend polynomially on the coefficients of $P$. However, one can be much more precise, using work of Macaulay, Hartshorne, Gotzmann, and Bayer, as follows. For a sequence $m_{0} \geq \cdots \geq m_{s}>0$ of positive integers, consider the degree $s$ numerical polynomial in $z$ :

$$
g\left(m_{0}, \ldots, m_{s} ; z\right)=\sum_{i=0}^{s}\left(\begin{array}{c}
z+i \\
i+1
\end{array}\right)-\left(\begin{array}{c}
z+i-m_{i} \\
i+1
\end{array}\right) .
$$

It can be shown that the Hilbert polynomial of any projective scheme $Z$ can be written uniquely in the form $g\left(m_{0}, \ldots, m_{s} ; z\right)$ for $s=\operatorname{dim}(Z)$ and suitable integers $m_{0} \geq m_{1} \geq$ $\cdots \geq m_{s}>0$. Furthermore, Gotzmann's regularity theorem states that $\mathcal{O}_{Z}$ is $\left(m_{0}-1\right)$ regular and $\mathcal{I}_{Z}$ is $m_{0}$-regular $[1,2,3]$.

This bound on the regularity of a subscheme is best possible, as can be verified by considering the "lexicographic" monomial ideal corresponding to a given numerical polynomial. By this we mean the following. Let $P(z)$ be written in the form $g\left(m_{0}, \ldots, m_{s} ; z\right)$. Consider the ideal $\mathcal{L}$ generated by the $\left(\begin{array}{c}n+m_{0} \\ n\end{array}\right)-P\left(m_{0}\right)$ first monomials in $H^{0}\left(\mathcal{O}_{\boldsymbol{P}^{n}}\left(m_{0}\right)\right)$, taken in the lexicographic order. Then one may show that the last of these monomials is not in the saturation of the ideal generated by the others. Hence the associated ideal sheaf is not generated by its sections of degree $m_{0}-1$.

The regularity bound gets large very quickly. For example, for the polynomial $P(z)=$ $(z+1)^{3}$, corresponding to the Segre imbedding of $\boldsymbol{P}^{1} \times \boldsymbol{P}^{1} \times \boldsymbol{P}^{1}$ in $\boldsymbol{P}^{7}$, we may compute that $m_{0}=3216$, and for $P(z)=(z+1)^{4}$, we get $m_{0}=141193125854001740$. This indicates that the Hilbert scheme is quite a beast, even for quite innocent looking subschemes!

R e mark 4.6. There is a similar statement to Proposition 4.3 (with the same proof) if instead of ideal sheaves one considers subsheaves on a fixed coherent sheaf $\mathcal{F}$ on $\boldsymbol{P}^{n}$, 
specializing to the above if we take $\mathcal{F}=\mathcal{O}_{\boldsymbol{P}^{n}}$. This can be used in proving that the "Quot" functor (see Section 9) is representable.

5. Base change. This paragraph is mostly extracted from [11]. We need some information on base change in the non-flat case. Suppose we have a diagram

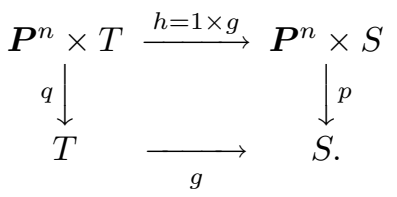

If $\mathcal{F}$ is a coherent sheaf on $\boldsymbol{P}^{n} \times S$, there are base change maps

$$
b_{i}: g^{*} R^{i} p_{*} \mathcal{F} \rightarrow R^{i} q_{*} h^{*} \mathcal{F} .
$$

The theorems on cohomology and base change in [6] deal only with the case where $\mathcal{F}$ is assumed flat over $S$. But even without this assumption, $b_{i}$ are isomorphisms if we replace $\mathcal{F}$ by $\mathcal{F}(m)$ for a sufficiently large $m$. Of course, then the higher direct images are zero, so the only interesting isomorphism is $b_{0}$.

Proposition 5.1. Let the situation be as above. Then there exists an $m_{0}$ such that for all $m \geq m_{0}$, the base change map $b_{0}: g^{*} p_{*} \mathcal{F}(m) \rightarrow q_{*} h^{*} \mathcal{F}(m)$ is an isomorphism.

Proof. This is local on $T$, so we may assume that $S=\operatorname{Spec}(A)$ and $T=\operatorname{Spec}(B)$ are affine. Recall the correspondence between coherent sheaves on $\boldsymbol{P}_{A}^{n}$ and graded modules over $A\left[X_{0}, \ldots, X_{n}\right]$. To a sheaf $\mathcal{F}$ there is associated the graded module $\bigoplus_{m} p_{*} \mathcal{F}(m)$, and to a graded module $M$ there is associated a coherent sheaf $\widetilde{M}$. This correspondence is 1-1 except that we may truncate the graded module from below wherever we want. The assertion of the proposition is just the fact that the maps in this 1-1 correspondence are compatible with tensor products $-\otimes_{A} B$.

Remark 5.2. Note that, a priori, $m_{0}$ depends on $g: T \rightarrow S$ as well as $\mathcal{F}$. Later we shall see that in fact $m_{0}$ can be chosen to depend only on $\mathcal{F}$.

We know from [6] that if $S$ is reduced and connected, then the sheaf $\mathcal{F}$ above is flat over $S$ if and only if all geometric fibers of $\mathcal{F}$ over $S$ have the same Hilbert polynomial. We need a similar criterion if the case of a general base $S$ :

Proposition 5.3. A coherent sheaf $\mathcal{F}$ on $\boldsymbol{P}_{S}^{n}$ is flat over $S$ if and only if there exists an $m_{0}$ such that $p_{*} \mathcal{F}(m)$ is locally free for all $m \geq m_{0}$.

Pr o of. If $\mathcal{F}$ is flat, this follows from standard base change theorems [6]. Conversely, if such an $m_{0}$ exists, let $M=\bigoplus_{m \geq m_{0}} p_{*} \mathcal{F}(m)$ be the associated graded $\mathcal{O}_{S}$-module. (We may assume that $S$ is affine.) Then $M$ is flat over $S$. Recall now how the functor $M \mapsto \widetilde{M}$ works: Over a suitable open affine in $\boldsymbol{P}_{S}^{n}$, the sheaf $\widetilde{M}$ is obtained from $M$ by first localizing in a variable $X_{i}$ and then extracting the direct summand of degree 0. Clearly these operations preserve flatness. Since $\widetilde{M}=\mathcal{F}$, we are done. 
6. Fitting ideals. Let $S$ be a scheme, and let $f: \mathcal{E}_{1} \rightarrow \mathcal{E}_{0}$ be a morphism of locally free sheaves of finite ranks $e_{1}$ and $e_{0}$, respectively. Locally, $f$ can be represented by an $e_{0} \times e_{1}$ matrix $m$ with entries in $\mathcal{O}_{S}$. The ideal generated by the minors of $m$ of a given size is independent of the choice of $m$, and globalizes to an ideal which in fact turns out to depend only on the cokernel of $f$.

Definition 6.1. Let $r$ be an integer. The $r$-th Fitting ideal $F_{r}(f)$ of $f$ is the image of the map

$$
\wedge^{e_{0}-r} \mathcal{E}_{1} \otimes \wedge^{e_{0}-r} \mathcal{E}_{0}^{\vee} \rightarrow \mathcal{O}_{S}
$$

induced by the map $\wedge^{e_{0}-r} f: \wedge^{e_{0}-r} \mathcal{E}_{1} \rightarrow \wedge^{e_{0}-r} \mathcal{E}_{0}$. If $r \geq e_{0}$ we agree that $F_{r}(f)=\mathcal{O}_{S}$, if $r<0$ then $F_{r}(f)=0$.

If $\mathcal{F}$ is a coherent sheaf on $S$, we define the $r$-th Fitting ideal $F_{r}(\mathcal{F})$ of $\mathcal{F}$ to be the $r$-th Fitting ideal of a locally free presentation of $\mathcal{F}$.

We need to verify that the Fitting ideal so defined is independent of the choice of presentation. So assume that $f$ is a presentation of $\mathcal{F}$. Note that the formation of Fitting ideals commutes with arbitrary base change on $S$. In particular, since both $F_{r}(f)$ and $\mathcal{F}=\operatorname{Coker}(f)$ localize well, we may assume that $S$ is the spectrum of a local $\operatorname{ring} A$ and that the $\mathcal{E}_{i}$ are free $A$-modules. Let $g: A^{n} \rightarrow A^{m}$ be a minimal presentation of $\mathcal{F}$. Then there exists a commutative diagram of $A$-modules:

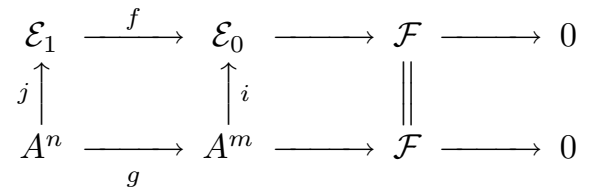

where $i$ and $j$ are split monomorphisms. It follows that $F_{r}(f)=F_{r}(g)$, and this proves that $F_{r}(f)$ only depends on $\mathcal{F}$.

Proposition 6.2. Let $\mathcal{F}$ be a coherent sheaf on $S$, and let $r$ be an integer. Then $\mathcal{F}$ is locally free of rank $r$ if and only if $F_{r-1}(\mathcal{F})=0$ and $F_{r}(\mathcal{F})=\mathcal{O}_{S}$.

Proof. Necessity is immediate. For sufficiency, we may again assume that $S=$ $\operatorname{Spec}(A)$ for a local ring $A$. Let $f: A^{n} \rightarrow A^{m}$ be a presentation of $\mathcal{F}$. Since $F_{r}(\mathcal{F})=A$ and $A$ is local, there is an invertible $(m-r) \times(m-r)$ minor of $f$. The corresponding invertible submatrix allows splitting off part of the presentation, and we are left with a presentation $g: A^{n-m+r} \rightarrow A^{r}$ of $\mathcal{F}$. Since $F_{r-1}(\mathcal{F})=0, g=0$ and we are done.

Corollary 6.3. Let $\mathcal{F}$ be a coherent sheaf on $S$, and let $r$ be an integer. Let $S_{r}(\mathcal{F})$ be the locally closed subscheme $V\left(F_{r-1}(\mathcal{F})\right)-V\left(F_{r}(\mathcal{F})\right)$ of $S$. Then for any morphism $g: T \rightarrow S$, the pullback $\mathcal{F}_{T}=g^{*}(\mathcal{F})$ is locally free of rank $r$ if and only if $g$ factors through the inclusion $S_{r}(\mathcal{F}) \subseteq S$.

P r o of. Apply Proposition 6.2 to $g^{*} \mathcal{F}$, noting that Fitting ideals commute with base change.

7. Flattening stratifications. In the previous section we essentially studied the question: Given a coherent sheaf $\mathcal{F}$ on $S$, what is the condition on an $S$-scheme $T$ that $\mathcal{F}_{T}$ be locally free? More generally, we may ask for flatness in cases where $\mathcal{F}$ is not coherent on $S$, but rather on a projective $S$-scheme. To be precise, suppose that we are 
in the situation (5.1), with $\mathcal{F}$ a coherent sheaf on $\boldsymbol{P}_{S}^{n}$. Even if $\mathcal{F}$ is not flat over $S$, it might happen that $\mathcal{F}_{T}=(1 \times g)^{*} \mathcal{F}$ is flat over $T$.

Definition 7.1. A flattening stratification for $\mathcal{F}$ over $S$ is finite disjoint collection $\left\{S_{i}\right\}$ of locally closed subschemes of $S$, the set-theoretic union of which is $S$, with the following property:

$$
(1 \times g)^{*} \mathcal{F} \text { is flat } \Longleftrightarrow \text { each } g^{-1} S_{i} \text { is open and closed in } T .
$$

If $T$ is connected, this means that $(1 \times g)^{*} \mathcal{F}$ is flat if and only if $T \rightarrow S$ factors through one of the $S_{i}$. The $S_{i}$ are called flattening strata of $\mathcal{F}$. A flattening stratification is not unique in general, but each connected component of a flattening stratum is a maximal locally closed connected subscheme of $S$ with respect to the property that the restriction $\mathcal{F}_{S_{i}}$ is flat. Hence we could have uniqueness of the flattening stratification if we imposed the condition that all strata be connected. However, this is not the most useful choice in general, and in the following proposition we lump together connected strata according to Hilbert polynomials.

Proposition 7.2. Let $\mathcal{F}$ be a coherent sheaf on $\boldsymbol{P}_{S}^{n}$. There exists a flattening stratification $\left\{S_{P}\right\}$ for $\mathcal{F}$, indexed by numerical polynomials $P$, such that for all $g: T \rightarrow S$, we have

$\mathcal{F}_{T}$ is $T$-flat with Hilbert polynomial $P \Longleftrightarrow g$ factors as $T \rightarrow S_{P} \rightarrow S$.

Proof. Look at the case $n=0$ first, i.e., $\mathcal{F}$ is a coherent sheaf on $S$ itself. Then the $S_{r}(\mathcal{F})$ form a flattening stratification, by Corollary 6.3. For dealing with the general case, we need the following

Lemma 7.3. Let $\mathcal{F}$ be a coherent sheaf on $\boldsymbol{P}_{S}^{n}$. Then there exists a nonempty open subset $U \subseteq S_{\text {red }}$ such that $\mathcal{F}_{U}$ is flat over $U$.

Note that unless $S$ is generically reduced, we may not be able to find such $U$ open in $S$ itself: consider for example the case where $n=0$ and $\mathcal{F}=\mathcal{O}_{S} / \mathcal{N}$, where $\mathcal{N}$ is the nilradical of $\mathcal{O}_{S}$.

Proof. Without loss of generality we may assume that $S$ is affine, reduced, and irreducible. Cover $\boldsymbol{P}_{S}^{n}$ by standard open affine sets $D_{i}=D_{+}\left(X_{i}\right)$ as usual, and let $\mathcal{F}_{i}$ be the restriction of $\mathcal{F}$ to $D_{i}$. If $U_{i} \subseteq S$ is an open subset such that $\mathcal{F}_{i}$ is flat over $U_{i}$, then $\mathcal{F}_{U}$ is flat over $U=U_{0} \cap \ldots \cap U_{n}$. Hence the lemma follows from the following pure algebra statement.

Proposition 7.4. Let $A \subseteq B$ be a finitely generated extension of noetherian integral domains, and let $F$ be a finitely generated $B$-module. Then there exists a non-zero $f \in A$ such that $F_{f}=F \otimes_{A} A_{f}$ is a flat $A$-module.

Proof. $F$ admits a finite filtration by submodules $F_{i}$ such that $F_{i} / F_{i+1} \simeq B / P$ for some prime ideal $P$ of $B$. It suffices therefore to prove the proposition in the special case $F=B / P$. If $P \cap A \neq 0$, then we may find a non-zero $f \in P \cap A$, and then $F_{f}=0$, which is flat. Thus we may assume that $P \cap A=0$. Dividing out by $P$, we may assume that $P=0$.

Let $K$ be the fraction field of $A$. By Noether's normalization lemma, there exist elements $b_{1}, \ldots, b_{r}$ in $B \otimes_{A} K$, algebraically independent over $K$, such that $B \otimes_{A} K$ is a 
finitely generated module over the polynomial ring $K\left[b_{1}, \ldots, b_{r}\right]$. For a suitable common denominator $a \in A$, we can assume that the $b_{i}$ are in $B_{a}$ and that $B_{a}$ is finite over $C:=A_{a}\left[b_{1}, \ldots, b_{r}\right]$. If the rank of $B_{a}$ as $C$-module is $t$, we may choose a $C$-linear map $\phi: C^{t} \rightarrow B_{a}$ such that $\phi$ is injective and has cokernel of dimension less than $\operatorname{dim}(B)$. By induction on the dimension, $\operatorname{Coker}(\phi)$ is flat over $A_{c}$ for some nonzero element $c \in A$. Then $B_{a c}$ is flat over $A_{a c}$.

COROLlary 7.5. There is a finite set of locally closed reduced subschemes $Y_{i}$ of $S$ such that their set-theoretic union is $S$ and such that $\mathcal{F}_{Y_{i}}$ is flat over $Y_{i}$ for all $i$. In particular, there is only a finite number of distinct Hilbert polynomials for the various geometric fibers $\mathcal{F}_{s}$ for $s \in S$, and we may, if necessary after collecting all $Y_{i}$ with the same Hilbert polynomials in the fibers, index the $Y_{i}$ by Hilbert polynomials and write $Y_{P}$ instead.

We may now complete the proof of Proposition 7.2. By Proposition 5.1, there exists an $m_{0}$ such that all the fibers of $\mathcal{F}$ are $m_{0}$-regular. (First apply Proposition 5.1 to each base change $Y_{P} \rightarrow S$, and find an $m_{0}^{\prime}$ as in that proposition that works for all the $Y_{P}$. Then apply Serre's theorem and standard base change arguments for flat families to ensure the vanishing of $H^{i}\left(\mathcal{F}_{y}(\ell)\right)$ for $i>0$ and large $\ell$, and $y \in Y_{P}$ a geometric point.)

Therefore, the Hilbert polynomial of a given fiber $\mathcal{F}_{s}$ is determined by the numbers $h^{0}\left(\mathcal{F}_{s}(m)\right)$ for $m_{0} \leq m \leq m_{0}+n$. For each $m \geq m_{0}+n$, put $\mathcal{M}_{m}=\bigoplus_{i=m_{0}}^{m} p_{*} \mathcal{F}(i)$. It now follows that the Hilbert polynomial of the fibers is constant on each flattening stratum of $\mathcal{M}_{m}$, for any $m \geq m_{0}+n$. As $m$ grows, the flattening strata of $\mathcal{M}_{m}$ form descending sequences of locally closed subschemes of $S$ with fixed support $Y_{P}$. Such sequences are stationary. Hence for $m$ large enough, by Proposition 5.3, the flattening strata for $\mathcal{M}_{m}$ are also flattening for $\mathcal{F}$.

Remark 7.6. For $m \gg 0$, the integers $P(m)$ are distinct as $P$ runs through the finite set of Hilbert polynomials. In conjunction with the above proof, this shows that the flattening stratification for $p_{*} \mathcal{F}(m)$ is also a flattening stratification for $\mathcal{F}$, if $m$ is large enough.

\section{Existence of the Hilbert scheme.}

THEOREM 8.1 (Grothendieck). Assume that $X$ is projective over $S$ and $P$ is a numerical polynomial. Then $\mathrm{Hilb}_{X / S}^{P}$ exists and is projective over $S$.

Proof. First step: we may assume that $X=\boldsymbol{P}_{S}^{n}$. Indeed, fix a closed imbedding $X \subseteq \boldsymbol{P}_{S}^{n}$ over $S$, and assume that $H=\operatorname{Hilb}_{\boldsymbol{P}_{S}^{n} / S}^{P}$ exists. Let $\mathcal{Z} \subseteq \boldsymbol{P}_{H}^{n}$ be the universal subscheme. Fix a sufficiently large integer $m$, and consider the zero locus of the composed map $\alpha$ of sheaves on $H$ :

$$
\alpha:\left(p_{*} \mathcal{I}_{X}(m)\right)_{H} \rightarrow\left(p_{*} \mathcal{O}_{\boldsymbol{P}_{S}^{n}}(m)\right)_{H}=\pi_{*} \mathcal{O}_{\boldsymbol{P}_{H}^{n}}(m) \rightarrow \pi_{*} \mathcal{O}_{\mathcal{Z}}(m)
$$

Here $p: \boldsymbol{P}_{S}^{n} \rightarrow S$ and $\pi: \boldsymbol{P}_{H}^{n} \rightarrow H$ are the natural maps. For a $T$-point $t: T \rightarrow H$, it is fairly clear that $\mathcal{Z}_{T} \subseteq X_{T}$ if and only if $t^{*}(\alpha)=0$. Therefore the zero locus of $\alpha$, defined by the appropriate Fitting ideal of $\operatorname{Coker}(\alpha)$, represents the Hilbert functor of $X / S$.

Second step: we may assume that we are in the absolute case, i.e., that $S=\operatorname{Spec}(\boldsymbol{Z})$. In general, if $S^{\prime} \rightarrow S$ is any base change and if $\operatorname{Hilb}_{X / S}^{P}$ exists, then $\operatorname{Hilb}_{X \times{ }_{S} S^{\prime} / S^{\prime}}^{P}=$ 
$\left(\operatorname{Hilb}_{X / S}^{P}\right) \times{ }_{S} S^{\prime}$. This is straightforward. Thus it remains to be proved that the Hilbert scheme of $\boldsymbol{P}^{n}=\boldsymbol{P}_{Z}^{n}$ exists.

Third step: to imbed the Hilbert functor into a Grassmann functor. Fix $m_{0}=m_{0}(P)$ as in Proposition 4.3. Then for any scheme $T$ and flat family of subschemes $Z \subseteq \boldsymbol{P}_{T}^{n}$ with Hilbert polynomial $P$ in the fibers, the induced map $H^{0}\left(\mathcal{O}_{\boldsymbol{P}^{n}}\left(m_{0}\right)\right)_{T} \rightarrow \operatorname{pr}_{T *} \mathcal{O}_{Z}\left(m_{0}\right)$ is surjective and the second sheaf locally free of rank $P\left(m_{0}\right)$. But this means that we get a $T$-point of the Grassmann variety $\operatorname{Grass}^{P\left(m_{0}\right)}\left(H^{0}\left(\mathcal{O}_{\boldsymbol{P}^{n}}\left(m_{0}\right)\right)\right)$. By base change theorems again, this is functorial in $T$, giving rise to a morphism of functors:

$$
\phi: \underline{\operatorname{Hilb}}_{\boldsymbol{P}^{n}}^{P} \rightarrow \underline{\operatorname{Grass}}^{P\left(m_{0}\right)}\left(H^{0}\left(\mathcal{O}_{\boldsymbol{P}^{n}}\left(m_{0}\right)\right)\right) .
$$

The morphism $\phi$ is injective. To see this, let $Z \subseteq \boldsymbol{P}_{T}^{n}$ correspond to an element $[Z] \in$ $\underline{\text { Hilb }}_{\boldsymbol{P}^{n}}^{P}(T)$, with ideal sheaf $\mathcal{I}_{Z}$. Then $\phi([Z])$ determines the following short exact sequence:

$$
0 \rightarrow \operatorname{pr}_{T *} \mathcal{I}_{Z}\left(m_{0}\right) \rightarrow H^{0}\left(\mathcal{O}_{\boldsymbol{P}^{n}}\left(m_{0}\right)\right)_{T} \rightarrow \operatorname{pr}_{T *}\left(\mathcal{O}_{Z}\left(m_{0}\right)\right) \rightarrow 0
$$

Composing the inclusion here with the pullback of the natural surjection

$$
H^{0}\left(\mathcal{O}_{\boldsymbol{P}^{n}}\left(m_{0}\right)\right)_{\boldsymbol{P}^{n}} \rightarrow \mathcal{O}_{\boldsymbol{P}^{n}}\left(m_{0}\right),
$$

we obtain, by base change, maps on $\boldsymbol{P}^{n} \times T$ :

$$
\operatorname{pr}_{T}{ }^{*} \operatorname{pr}_{T *} \mathcal{I}_{Z}\left(m_{0}\right) \rightarrow H^{0}\left(\mathcal{O}_{\boldsymbol{P}^{n}}\left(m_{0}\right)\right)_{\boldsymbol{P}^{n} \times T} \rightarrow \mathcal{O}_{\boldsymbol{P}^{n}}\left(m_{0}\right)_{\boldsymbol{P}^{n} \times T}
$$

where, by Proposition $4.2(3)$ for $\mathcal{I}_{Z}$, the image of the composed map is $\mathcal{I}_{Z}\left(m_{0}\right)$. Hence $Z$ is determined by $\phi([Z])$.

Fourth step: to show that the image of $\phi$ is a locally closed subfunctor of the Grassmann functor. Denote by $G$ the Grassmann variety

$$
G=\operatorname{Grass}^{P\left(m_{0}\right)}\left(H^{0}\left(\mathcal{O}_{\boldsymbol{P}^{n}}\left(m_{0}\right)\right)\right),
$$

and let

$$
0 \rightarrow R \rightarrow H^{0}\left(\mathcal{O}_{\boldsymbol{P}^{n}}\left(m_{0}\right)\right)_{G} \rightarrow Q \rightarrow 0
$$

be the universal exact sequence on $G$. Taking the leftmost map here and the tautological map

we obtain a map on $\boldsymbol{P}^{n} \times G$ :

$$
H^{0}\left(\mathcal{O}_{\boldsymbol{P}^{n}}\left(m_{0}\right)\right)_{\boldsymbol{P}^{n}} \rightarrow \mathcal{O}_{\boldsymbol{P}^{n}}\left(m_{0}\right),
$$

$$
\operatorname{pr}_{G}{ }^{*} R \rightarrow H^{0}\left(\mathcal{O}_{\boldsymbol{P}^{n}}\left(m_{0}\right)\right)_{\boldsymbol{P}^{n} \times G} \rightarrow \mathcal{O}_{\boldsymbol{P}^{n}}\left(m_{0}\right)_{\boldsymbol{P}^{n} \times G}
$$

where the image of the composed map is $\mathcal{I}_{W}\left(m_{0}\right)$ for some closed subscheme $W \subseteq \boldsymbol{P}_{G}^{n}$. Now consider the flattening stratum $G_{P}$ for the coherent sheaf $\mathcal{O}_{W}$. I claim that the image of $\phi$ is exactly $h_{G_{P}}$. Indeed, for any family $[Z] \in \underline{\operatorname{Hilb}}_{\boldsymbol{P}^{n}}^{P}(T)$, we have already shown above that the pullback of $W$ under the induced map $T \rightarrow G$ is just $Z$ again. Since this is flat over $T$, we have a factorization $T \rightarrow G_{P} \rightarrow G$. This shows that the image of $\phi$ is contained in $h_{G_{P}}$. On the other hand, the restriction $W_{G_{P}}$ is flat with Hilbert polynomial $P$, hence gives rise to a $G_{P}$-valued point of the Hilbert functor, or equivalently, a morphism $\psi: h_{G_{P}} \rightarrow \underline{\operatorname{Hilb}}_{P^{n}}^{P}$. Clearly $\psi$ is a two-sided inverse to $\phi$, which completes step four.

It follows by Proposition 2.12 that $\operatorname{Hilb}_{\boldsymbol{P}^{n}}^{P}$ exists and is quasiprojective over $\boldsymbol{Z}$.

The fifth and final step is to show that $\operatorname{Hilb}_{\boldsymbol{P}^{n}}^{P}$ is complete, i.e., proper over $\operatorname{Spec}(\boldsymbol{Z})$. For this one uses the valuative criterion for completeness: Let $A$ be a discrete valuation ring and let $U \subseteq \bar{U}=\operatorname{Spec}(A)$ be the complement of the closed point. Suppose $u: U \rightarrow$ 
Hilb $_{\boldsymbol{P}^{n}}^{P}$ is given, corresponding to a subscheme $Z \subseteq \boldsymbol{P}^{n} \times U$ flat over $U$. Now simply let $\bar{Z} \subseteq \boldsymbol{P}_{\bar{U}}^{n}$ be the closure of $Z$. Then $\bar{Z}$ is flat over $\bar{U}$ [6], hence $u$ can be extended to $\bar{u}: \bar{U} \rightarrow \operatorname{Hilb}_{\boldsymbol{P}^{n}}^{P}$.

Remark 8.2. Hartshorne has proved that $\operatorname{Hilb}_{\boldsymbol{P}^{n}}^{P}$ is connected [5]. Not much is known about the number of irreducible components in general; for example for space curves, the problem has been studied for more than a century, and in spite of some progress, the classification of irreducible components seems completely out of reach. Even components where the general point corresponds to a nonsingular curve can be non-reduced, as was first observed by Mumford [10].

9. Variations. These variations are taken from Grothendiecks original paper [4] where he defines and constructs the Quot and Hilbert schemes.

Quot scheme. The first variation is the generalization to the Quot scheme. The setting is this: Let $X$ be an $S$-scheme and let $\mathcal{F}$ be a coherent sheaf on $X$. For any $S$-scheme $T$, put

$$
\text { Quot }_{\mathcal{F} / X / S}(T)=\left\{\text { coherent quotient sheaves } \mathcal{F}_{T} \rightarrow \mathcal{G} \text {, with } \mathcal{G} \text { flat over } T\right\},
$$

where as usual quotients are equal if their kernels coincide. Then we have the following theorem:

Theorem 9.1. Assume that $X \rightarrow S$ is projective, $X \subseteq \boldsymbol{P}_{S}^{n}, \mathcal{F}$ a coherent sheaf on $X$, and $P$ a numerical polynomial. Let $\underline{\text { uot }}_{\mathcal{F} / X / S}^{P}$ be the subfunctor of $\underline{\text { uot }}_{\mathcal{F} / X / S}$ corresponding to quotients with Hilbert polynomial $P$. Then $\underline{\mathrm{Quot}}_{\mathcal{F} / X / S}^{P}$ is represented by a projective $S$-scheme $\operatorname{Quot}_{\mathcal{F} / X / S}^{P}$.

Note that $\mathcal{F}$ needs not necessarily be flat over $S$. The proof is very similar to the one we have given for the Hilbert scheme. First, if $\mathcal{F}^{\prime} \rightarrow \mathcal{F}$ is a surjection, there is induced a closed imbedding Quot $\underline{\mathcal{F} / X / S} \rightarrow \underline{\text { Quot }}_{\mathcal{F}^{\prime} / X / S}$ of functors, so it suffices to prove that $\underline{\operatorname{Quot}}_{\mathcal{F} / X / S}^{P}$ is representable in the special case where $\mathcal{F}=N \mathcal{O}_{\boldsymbol{P}_{S}^{n}}(-m)$ for some integer $m$. Twisting everything by $m$ and replacing $P$ by the polynomial $z \mapsto P(z+m)$ we may assume that $\mathcal{F}=N \mathcal{O}_{\boldsymbol{P}_{S}^{n}}$. From there on everything goes more or less exactly as in the Hilbert scheme case, by finding a regularity bound in terms of $P$ and an imbedding of the Quot functor in the Grassmann functor given by taking direct image of a twist higher than the regularity.

If $\mathcal{F}=\mathcal{O}_{X}$, we recover the Hilbert scheme: $\operatorname{Quot}_{\mathcal{O}_{X} / X / S}^{P}=\operatorname{Hilb}_{X / S}^{P}$.

In another direction, suppose that $X=S$. Then any Hilbert polynomial is a constant, and a quotient is flat if and only if it is locally free. So we recover the Grassmannian again, $\operatorname{Quot}_{\mathcal{F} / S / S}^{r}=\operatorname{Grass}^{r}(\mathcal{F})$ as $S$-schemes. Note therefore that the Grassmannian exists even when $\mathcal{F}$ is not locally free. It is essential here that we consider quotients and not subsheaves, of course; this is one of the advantages of Grothendieck's contravariant constructions, like $\boldsymbol{P}(\mathcal{F})$.

Quasi-projective case. Suppose $U \subseteq X$ is an open subscheme of some projective scheme $X$ over $S$. Consider the following subfunctor $h$ of the Hilbert functor of $X / S$ :

$$
h(T)=\left\{[Z] \in \operatorname{Hilb}_{X / S}^{P}(T) \mid Z \subseteq U \times_{S} T \subseteq X \times{ }_{S} T\right\} .
$$


Then $h$ is represented by an open subscheme of $\operatorname{Hilb}_{U / S}^{P} \subseteq \operatorname{Hilb}_{X / S}^{P}$. This depends actually only on the quasi-projective $S$-variety $U$, as it coincides with the functor of subschemes of $U \times{ }_{S} T$ which are proper (or equivalently, projective) over $S$. For example, we have a Hilbert scheme for closed subschemes of $\boldsymbol{A}^{n}$ of fixed finite length.

Sections and morphisms. Let $f: X \rightarrow Y$ be a separated $S$-morphism. Consider the functor

$$
\prod_{Y / S}(X / Y)(T)=\left\{\text { morphisms } g: Y_{T} \rightarrow X_{T} \text { such that } f_{T} \circ g=1_{Y_{T}}\right\} .
$$

Clearly, an element of $\prod_{Y / S}(X / Y)(T)$ is equivalent to the datum of a closed subscheme of $X_{T}$ mapping isomorphically to $Y_{T}$ under $f_{T}$. Thus if $Y$ is flat over $S$, then $\prod_{Y / S}(X / Y)$ is an open subfunctor of $\underline{\operatorname{Hilb}}_{X / S}$. It follows that

THEOREM 9.2. If $Y$ is flat and proper over $S$, and $X$ is quasi-projective over $S$, then $\prod_{Y / S}(X / Y)$ is represented by an open subscheme of $\mathrm{Hilb}_{X / S}$.

In the special case when $X=Y \times_{S} Z$ and $f$ is the first projection, a section of $f$ over $Y_{T}$ is nothing but a morphism $Y \rightarrow Z$. Hence

THEOREM 9.3. If $Y$ is projective and flat over $S$, and $Z$ is quasi-projective over $S$, the functor

$$
T \mapsto\left\{\text { set of } S \text {-morphisms } Y_{T} \rightarrow Z_{T}\right\}
$$

is representable by an $S$-scheme $\operatorname{Hom}_{S}(Y, Z) \subseteq \operatorname{Hilb}_{Y \times_{S} Z / S}$. The subfunctor of isomorphisms $Y_{T} \rightarrow Z_{T}$ is represented by an open subscheme $\operatorname{Isom}_{S}(Y, Z) \subseteq \operatorname{Hom}_{S}(Y, Z)$.

There are many other variations as well that one may build from Hilbert schemes. For example, one might want to study nested pairs $Z \subseteq Y$ of subschemes of $X$, or more general collections of subschemes of several schemes with morphisms between them on so on. One example which has been useful is the parameter space for morphisms $f: \boldsymbol{P}^{1} \rightarrow X$ taking a given finite collection of points $P_{i} \in \boldsymbol{P}^{1}$ to specified subschemes $Y_{i} \subseteq X$. This space plays a part in areas such as the Mori minimal model program as well as in the study of mirror symmetry and Calabi-Yau manifolds.

10. Local structure of the Hilbert scheme. We will not attempt to go into deformation theory in any detail, except to prove Grothendieck's formula for the tangent space of the Hilbert scheme.

THEOREM 10.1. Let $X$ be an algebraic scheme over a field $k$, and assume that the Hilbert scheme $H=$ Hilb $_{X / k}$ exists (for example, that $X$ is projective over $k$ ). Let $[Z] \in H$ correspond to a subscheme $Z \subseteq X$. Then the Zariski tangent space of the Hilbert scheme $H$ at the point $[Z]$ is canonically isomorphic to $\operatorname{Hom}_{X}\left(\mathcal{I}_{Z}, \mathcal{O}_{Z}\right)$.

Proof. Let $D=\operatorname{Spec}(k[\epsilon])$ where $\epsilon^{2}=0$. There is a natural inclusion $i: \operatorname{Spec}(k) \rightarrow D$. As remarked earlier, the tangent space to $H$ at $[Z]$ can be identified with the set of $\xi \in h_{H}(D)$ such that the $i^{*}(\xi)=[Z]$. We will not go into all details in this proof, but be content with giving a description of both ways of the natural correspondence asserted in the theorem.

First, let $\widetilde{Z} \subseteq X \times D$ be a subscheme corresponding to a tangent vector $\xi$, i.e., it is flat over $D$ and restricts to $Z$ modulo $\epsilon$. We will construct a homomorphism $\phi_{\xi}: \mathcal{I}_{Z} \rightarrow \mathcal{O}_{Z}$. 
Tensoring together the exact sequences $0 \rightarrow k \stackrel{\epsilon}{\rightarrow} k[\epsilon] \stackrel{p}{\rightarrow} k \rightarrow 0$ and $0 \rightarrow \mathcal{I}_{\widetilde{Z}} \rightarrow$ $\mathcal{O}_{X} \otimes_{k} k[\epsilon] \rightarrow \mathcal{O}_{\widetilde{Z}} \rightarrow 0$ and using the flatness assumption, we get a commutative exact diagram

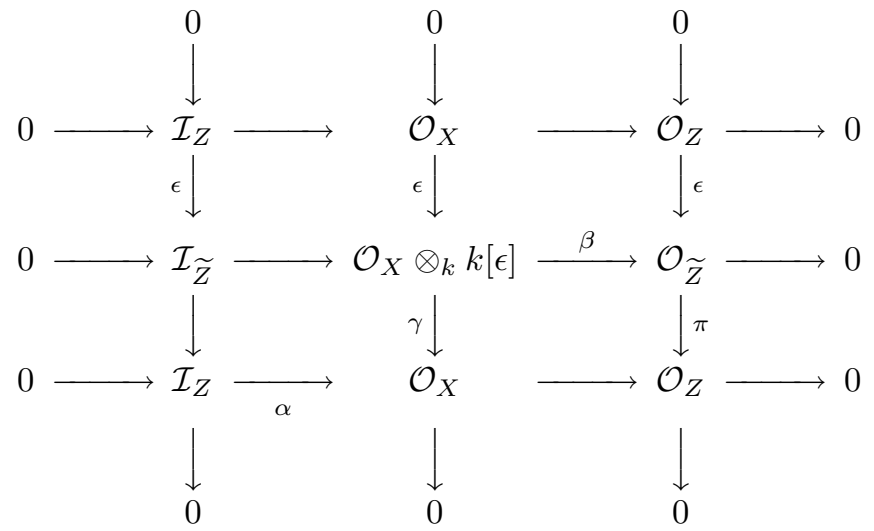

The inclusion $k \rightarrow k[\epsilon]$ induces a section $j: \mathcal{O}_{X} \rightarrow \mathcal{O}_{X} \otimes_{k} k[\epsilon]$ of $\gamma$. The composed map $\beta \circ j \circ \alpha$ clearly has image contained in the kernel of $\pi$, hence induces a map

$$
\phi_{\xi}: \mathcal{I}_{Z} \rightarrow \mathcal{O}_{Z} .
$$

Conversely, starting with an element $\phi \in \operatorname{Hom}_{X}\left(\mathcal{I}_{Z}, \mathcal{O}_{Z}\right)$, we can construct a lifting $\widetilde{Z}$ of $Z$ as follows: First cover $X$ by open affine subschemes $U$. Then $X \times D$ is covered by open affine subschemes $U \times D$, and it suffices to construct $\widetilde{Z}$ by glueing together its restrictions to these open affine sets. Let $U=\operatorname{Spec}(A)$ be an open affine of $X$, and let the ideal of $Z \cap U$ be generated by the ideal $I=\left(f_{1}, \ldots, f_{r}\right) \subseteq A$. Now construct an ideal $\widetilde{I} \subseteq \widetilde{A}=A \otimes_{k} k[\epsilon]$ in the following way: For each $i=1, \ldots, r$, let $g_{i} \in A$ be a lift of the element $\phi\left(f_{i}\right) \in A / I$, put $\widetilde{f}_{i}=f_{i}+\epsilon g_{i}$, and let $\widetilde{I}=\left(\widetilde{f}_{1}, \ldots, \widetilde{f}_{r}\right)$. Then one needs to check that the ideal $\widetilde{I}$ is independent of the choices made (of the generators $f_{i}$ and the liftings $g_{i}$ ) and that $\widetilde{A} / \widetilde{I}$ is flat over $k[\epsilon]$. We leave this check to the reader, as well as the verification that the various $\widetilde{I}$ taken together defines a subscheme $\widetilde{Z} \subseteq X \times D$ as required.

Finally, one verifies that the two constructions we have given are inverses to each other. This completes the proof.

Any homomorphism of $\mathcal{O}_{X}$-modules $\mathcal{I}_{Z} \rightarrow \mathcal{O}_{Z}$ factors uniquely through $\mathcal{I}_{Z} / \mathcal{I}_{Z}^{2}$. This sheaf is called the conormal sheaf of $Z$ in $X$, and its dual is called the normal sheaf $\mathcal{N}_{Z / X}$. If $Z$ is a local complete intersection in $X$, for example if both $X$ and $Z$ are nonsingular, the conormal and normal sheaves are locally free, and are called the conormal and normal bundle, respectively. Thus the first statement in the following theorem, which we state without proof, is just a rewrite of Theorem 10.1.

ThEOREM 10.2. The tangent space of the Hilbert scheme $H=\mathrm{Hilb}_{X / k}$ at the point $[Z]$ is isomorphic to $H^{0}\left(Z, \mathcal{N}_{Z / X}\right)$. If $Z$ is a local complete intersection in $X$, we have the inequalities

$$
h^{0}\left(\mathcal{N}_{Z / X}\right)-h^{1}\left(\mathcal{N}_{Z / X}\right) \leq \operatorname{dim}_{[Z]} H \leq h^{0}\left(\mathcal{N}_{Z / X}\right) .
$$

In particular, if $H^{1}\left(\mathcal{N}_{Z / X}\right)=0$, then $H$ is nonsingular at $[Z]$. More generally, if the first inequality is an equality, then $H$ is a local complete intersection at $[Z]$. 
Part of this is proved in [4], but the lower bound for the dimension is not there. For a complete proof of this theorem and similar theorems in much more general situations, see Laudal's work [8]. For a related case, the lower bound was proved by Mori [9]. The essential ingredient is a technique called obstruction calculus. For example, suppose that $\widetilde{Z}$ is a lifting of $Z \subseteq X$ to $k[\epsilon]=k[t] /\left(t^{2}\right)$ as in in the proof above. Then one may ask whether there $\widetilde{Z}$ can be further lifted to $k[t] /\left(t^{3}\right)$, and so on. The systematic study of such lifting problems is the domain of deformation theory. It turns out that there is a functorially defined element (called the obstruction) in some sort of cohomology group (obstruction group) which vanishes exactly when a lifting exists. Information bounding the size of the obstruction group is useful even when computing the actual obstructions is impractical. For example, the theorem above follows quite formally from general deformation theory $[8,4.2 .4]$ once we can establish that the obstruction group for the Hilbert scheme at a local complete intersection $Z \subseteq X$ is contained in $H^{1}\left(Z, \mathcal{N}_{Z}\right)$.

\section{References}

[1] D. Bayer, The division algorithm and the Hilbert scheme, Ph.D. thesis, Harvard University, May 1982.

[2] G. Gotzmann, Eine Bedingung für die Flachheit und das Hilbertpolynom eines graduierten Ringes, Math. Z. 158 (1978), 61-70.

[3] M. Green, Restrictions of linear series to hyperplanes, and some results of Macaulay and Gotzmann, in: Algebraic curves and projective geometry, Proceedings, Trento 1988, Lecture Notes in Math. 1389, Springer, Berlin, 1988.

[4] A. Grothendieck, Techniques de construction et théorémes d'existence en géométrie algébrique IV: Les schémas de Hilbert, Séminaire Bourbaki 221, 1960/61.

[5] R. Hartshorne, Connectedness of the Hilbert scheme, Inst. Hautes Études Sci. Publ. Math. 29 (1966), 261-309.

[6] R. Hartshorne, Algebraic Geometry, Graduate Texts in Math. 52, Springer, Berlin, Tokyo, New York, 1977.

[7] S. L. Kleiman, Geometry of grassmannians and applications to splitting bundles and smoothing cycles, Inst. Hautes Études Sci. Publ. Math. 36 (1969), 281-297, volume dedicated to Oscar Zariski.

[8] O. A. Laudal, Formal Moduli of Algebraic Structures, Lecture Notes in Math. 754 Springer, Berlin-Heidelberg-New York-London-Paris-Tokyo, 1979.

[9] S. Mori, Projective manifolds with ample tangent bundles. Ann. of Math. (2) 110 (1979), 593-606.

[10] D. Mumford, Further pathologies in algebraic geometry, Amer. J. Math. 84 (1962), 642648.

[11] D. Mumford, Lectures on Curves on an Algebraic Surface, Studies in Mathematics 59, Princeton University Press, 1968.

[12] D. Mumford, The red book of varieties and schemes, Lecture Notes in Math. 1358, Springer, Berlin-Heidelberg-New York-London-Paris-Tokyo, 1988.

[13] M. Schlessinger, Functors of Artin rings, Trans. Amer. Math. Soc. 130 (1968), 208-222. 